\title{
MAGNETIC INDUCTION MONITOR MEASUREMENTS OF BEAM SPATIAL CHARACTERISTICS IN TECHNOLOGICAL ELECTRON LINEAR ACCELERATORS
}

\author{
V.N. Boriskin, V.A. Gurin, A.N. Dovbnya, V.A. Popenko, L.V. Reprintsev, A.N. Savchenko, \\ D.L. Stepin, V.I. Tatanov, V.A. Shendrik, \\ NSC“Kharkov Institute of Physics\&Technology”, Ukraine
}

\begin{abstract}
A high-current two-section pulsed electron linear accelerator (ELA) "EPOS" has been in successful operation at "Accelerator" $\mathrm{R} \& \mathrm{D}$ Production Establishment, NSC KIPT, for a few years. Its main parameters are the current pulse length - $4 \mu$ s, the pulse current 0.4 to $1 \mathrm{~A}$, the pulse recurrence rate $-150 \mathrm{~Hz}$, the electron energy - up to $30 \mathrm{MeV}$. To provide a safe beam guiding, four-winding magnetic induction monitors were devised, making possible measurements of the position of beam current center relative to the accelerator axis with an error of $\pm 0.5 \mathrm{~mm}$ at the input and output of the second accelerating section [1]. At present, the "Accelerator" specialists have developed a compact two-section ELA "KUT-20", at the output of which a similar magnetic induction monitor is installed. Below, we present the results of bench calibration of the monitor as well as the experimental results of measuring-channel operation at the "EPOS" accelerator. The possibility of determining the second moment, which characterizes the difference of the beam cross section from its axially symmetric section, is demonstrated $[2,3]$
\end{abstract}

\section{MEASURING CHANNELS AND MONITORS}

The schematic diagram of the position monitor is shown in Fig. 1. The monitor comprises independent windings, the signals from which carry information on both the current and position of the beam. The windings are wound around a ferrite ring, which is $60 \mathrm{~mm}$ in inner diameter and $100 \mathrm{~mm}$ in outer diameter, the height being $30 \mathrm{~mm}$.
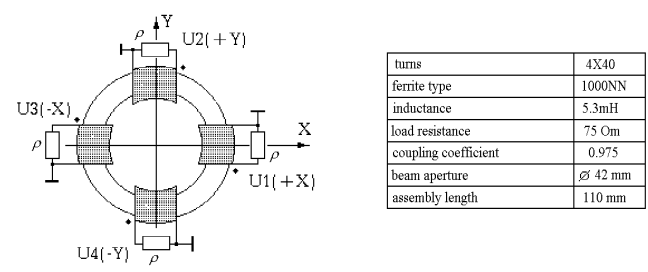

Figure 1: Circuit diagram and main design data of the beam position monitor. $\rho$ is the wave resistance of cable lines, $\mathrm{U}_{1}-\mathrm{U}_{4}$ are the matching load voltages.

The circuit used here provides rather high unipolar signals at monitor windings, and this makes their processing easier. To estimate horizontal $(\mathrm{H})$ and vertical
(V) beam center displacements from the monitor axis, we have used the following dimensionless functions:

$H=\left(U_{1}-U_{3}\right) /\left(U_{1}+U_{3}\right) \quad V=\left(U_{2}-U_{4}\right) /\left(U_{2}+U_{4}\right)$

Owing to the optimum configuration of the windings and the monitor screen design, the self-magnetic field of pair windings can be considered to be dipole [3]. Therefore, $\mathrm{H}$ and $\mathrm{V}$ will linearly depend on only one coordinate $\mathrm{X}=$ $a_{x} H, Y=a_{y} V$, where the coefficients $a_{x}$ and $a_{y}$ are calculated on calibration. To reduce the effect of $\mathrm{HF}$ noise, the integral value $T_{\text {int }}$ of the first half of $U$ signal from the monitor windings was substituted into (1).

Fig. 2 shows the skeleton diagram of one channel to monitor the beam position and the beam current value. The pulses from the windings of monitors loaded directly in the communication line go from the accelerator to the matched input of noise-balancing circuits at the control panel and then to the scaling amplifiers $\left(\mathrm{K}_{\mathrm{amp}}=3\right.$, front up to $0.1 \mu \mathrm{s})$. The low-frequency noise is suppressed by means of the isolating pulse transformer with an ungrounded primary winding, and the high-frequency noise is suppressed with the low-frequency filter having a cut-off frequency of $2 \mathrm{MHz}$. The signals from the amplifier outputs arrive at the input of the analog switching unit and then at the two-channel 8-digit analogdigital converter having a dynamic range of $2 \mathrm{~V}$, a 256 byte buffer storage and a clock rate of $10 \mathrm{MHz}$.

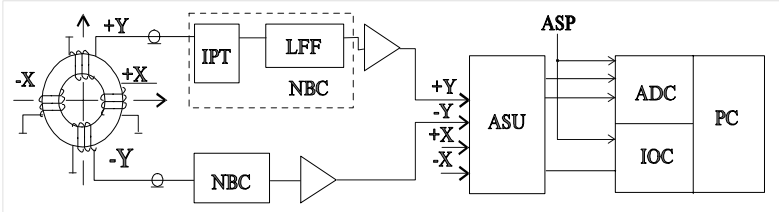

Figure 2: Block diagram of the channel to monitor ELA beam position and current. NBC - noise-balancing circuit, IPT - isolating pulse transformer, LFF - low-frequency filter, ASU - analog switching unit, ADC - analog-digital converter, IOC - input-output controller, ASP accelerator sync pulse.

Prior to its installation in the ELA, each monitor was subjected to calibration tests on a special bench. The bench included the beam current simulator, the metric head allowing the monitor to move in two coordinates with a set-on accuracy of $0.01 \mathrm{~mm}$, the automated measuring channel (see Fig. 2). 


\section{METHODS AND RESULTS OF MONITOR BENCH TESTS}

To simulate the electron beam, we have used one (Fig. 4a) or two (Figs. 4c, d) current-conducting strings, 0.2 $\mathrm{mm}$ across, stretched perpendicularly to the monitor plane. Along the strings, rectangular current pulses (Fig. $3 \mathrm{a}$ ), from 300 to $800 \mathrm{~mA}$ in the amplitude and $4 \mu \mathrm{s}$ in duration, were passed. As a result, induced signals (e.g., see Fig. 3b) were observed at the opposite windings of the monitor.

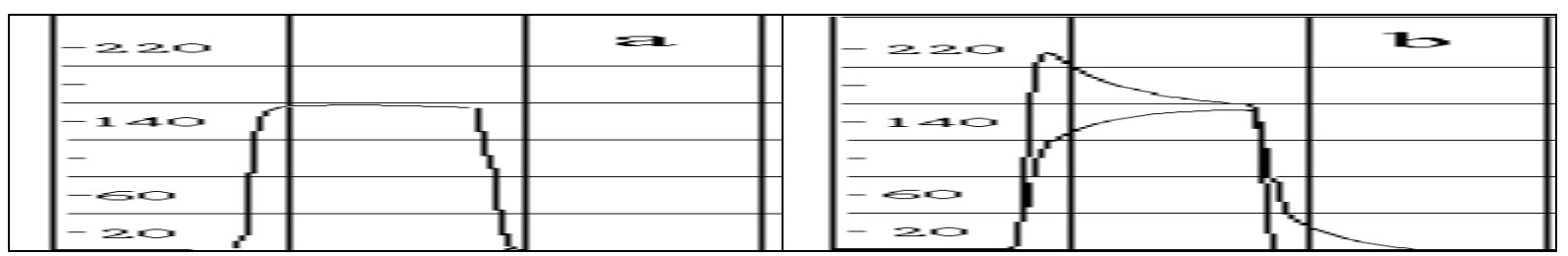

Figure 3: Shapes of current pulse in the string (a) and induced signals in monitor windings (b).

As a simplified mathematical model, we have used the known expression for the linear-current $(I)$ magnetic field vector [4]. If the angular dimensions of the signal winding are small, and the current flows in the vicinity of the coordinate origin $(r<<a)$, then, without including the constant factors, the field component $B$ along the circumference of radius $a$ (Fig. 4 a) will be $\dot{B} \approx[" I \times \dot{I}] \frac{\left(1-\frac{\dot{r} a}{a_{1}^{2}}\right)}{(\dot{a}-\dot{r})^{2}}$, this being equivalent to

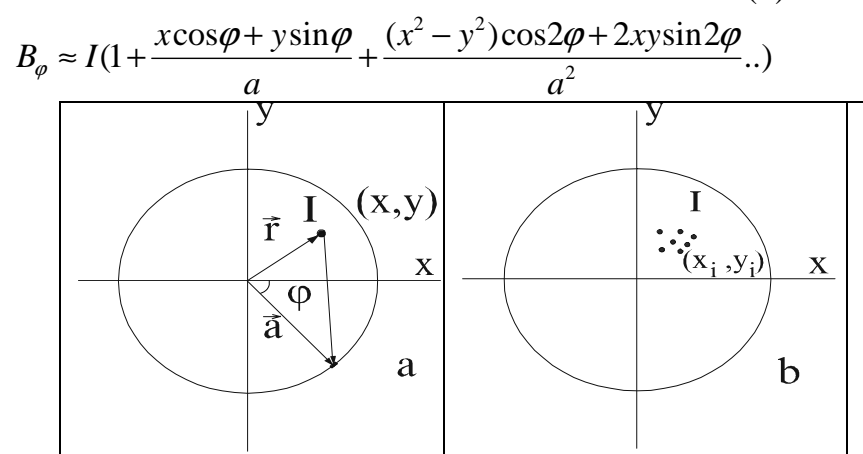

Having determined the $B_{\varphi}$ value for four points of the circumference $(\varphi=0, \pi / 2, \pi, 3 \pi / 2)$ and considering that $U$ is proportional to $d B / d t$, it can be shown that with neglection of small terms of the series in (2) we have $X \approx a H, Y \approx a V$. And for the electron beam (Fig. 4b), if $\overline{x^{2}}=\bar{x}^{2}+\sigma_{x}^{2}, \overline{y^{2}}=\bar{y}^{2}+\sigma_{y}^{2}$ one can derive the known expression [2] providing the estimate of the second moment of the beam cross-section:

$$
Q=\left(\sigma_{x}^{2}-\sigma_{y}^{2}\right)=a_{\sigma} D-\left(\bar{x}^{2}-\bar{y}^{2}\right)+q_{0},
$$

where $D=\left(U_{1}+U_{3}-U_{2}-U_{4}\right) / \sum_{1}^{4} U_{i}, \quad a_{\sigma \approx a^{2}}$.

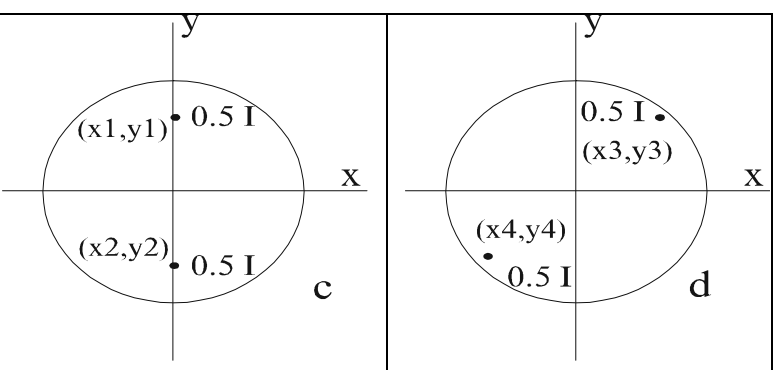

Figure 4: Examples of disposition of one (a), or two (c,d) strings, and also of the electron beam (b) in the plane of the monitor.

Of course, the given model does not take into account a number of important parameters (presence of the ferrite ring, angular size of windings, mutual induction of windings, etc.). Yet, it enables one to estimate the order of magnitude of $a_{x}, a_{y}$ and $a_{\sigma}$, and shows that $a_{x}{ }^{2}=a_{y}{ }^{2} \approx a_{\sigma}$, and, what is most important, shows the linearity of the functions $H(X), V(Y), D(Q)$ in the operating range of the In the calculations of $a_{x}, a_{y}$ and $a_{\sigma}$ an effort was made to take into account the above-mentioned parameters (see Table 1) for the toroidal core of inner radius $r_{l}$ and outer radius $r_{2}$. The version A corresponds to model (2), where the angular size of windings is $2 \chi=0$, and the radius of core is $r_{2} \rightarrow r_{1}=a$. The low table row gives the calculations for the ELA EPOS monitors $\left(T_{1}\right.$ and $T_{2}$ are the falloff constants of difference signal peaks in the calculation of $a_{x}$ and $a_{\sigma}$; the bracketed $a_{x}$ and $a_{\sigma}$ values were calculated from signal amplitudes). The exact coefficient values were determined using the test bench ( see Fig..5)

Table 1. Calculated $a_{x}, a_{y}$ and $a_{\sigma}$ for monitor models $\left(r_{l}=30 \mathrm{~mm}, r_{2}=50 \mathrm{~mm}, 2 \chi=\pi / 2, T_{\mathrm{int}}==2 \mu \mathrm{s}\right)$.

\begin{tabular}{|c|c|c|c|c|}
\hline Version & Calculation formula for $a_{x}=a_{y}$ & Calculation formula for $a_{\sigma}$ & $a_{x}=a_{y}(\mathrm{~mm})$ & $a_{\sigma}\left(\mathrm{mm}^{2}\right)$ \\
\hline $\mathrm{A}$ & $r_{1}$ & $\left(r_{1}\right)^{2}$ & 30 & 900 \\
\hline $\begin{array}{c}\mu=1, k_{c b}=0, \\
2 \chi=0, r_{1} \neq r_{2}\end{array}$ & $\frac{r_{1} \ln \left(r_{2} / r_{1}\right)}{\left(r_{2}-r_{1}\right) / r_{2}}$ & $\frac{2 r_{1}^{2} \ln \left(r_{2} / r_{1}\right)}{\left(r_{2}^{2}-r_{1}^{2}\right) / r_{2}^{2}}$ & 38 \\
\\
\hline
\end{tabular}




\begin{tabular}{|c|c|c|c|c|}
\hline $\begin{array}{c}\mu=1, k_{c b}=0, \\
2 \chi \neq 0, r_{1} \neq r_{2}\end{array}$ & $\frac{r_{1} \ln \left(r_{2} / r_{1}\right)}{((\sin (\chi)) / \chi)\left(\left(r_{2}-r_{1}\right) / r_{2}\right)}$ & $\frac{2 r^{2} \ln \left(r_{2} / r_{1}\right)}{((\sin (2 \chi)) / 2 \chi)\left(\left(r_{2}^{2}-r_{1}^{2}\right) / r_{2}^{2}\right)}$ & 42 & 2260 \\
\hline$\mu \triangleright>1, k_{c b}=0$, & $\left(\frac{r_{1} \pi}{4 \sin (\chi)}\right) \frac{T_{\mathrm{int}} / T_{1}}{1-\exp \left(-T_{\mathrm{int}} / T_{1}\right)}$ & $\left(\frac{r_{1}^{2} \pi}{2 \sin (2 \chi)}\right) \frac{T_{\mathrm{int}} / T_{2}}{1-\exp \left(-T_{\mathrm{int}} / T_{2}\right)}$ & $(33)$ & $(1410)$ \\
$2 \chi \neq 0, r_{1} \neq r_{2}$ & & & \\
\hline
\end{tabular}
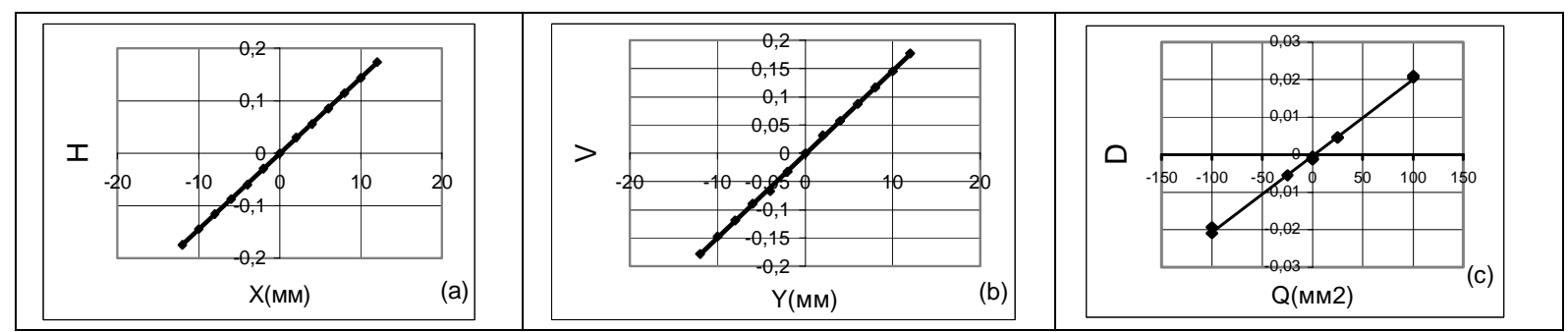

Figure 5: Observed dependences $\mathrm{H}(\mathrm{X}), \mathrm{V}(\mathrm{Y}), \mathrm{D}(\mathrm{Q})$ for the ELA KUT-20 monitor.

\section{MONITORING OF ELA EPOS BEAM PARAMETERS}

The automated monitoring system of the ELA EPOS [5] is equipped with the channels to monitor the spatial beam characteristics. During operation of high-current ELAs, the measurement channels are acted upon by external noise. In our case, there are generally two types of noise. These are high-frequency noise, which are modulated onto the top of the beam current pulse (Fig. 7) and low-frequency noise mainly due to the mains supply; it changes the signal amplitude from pulse to pulse. It should be noted that apart from HF noise, the shape of the current pulse top is also affected by the space-time structure of the beam; this is also seen in Fig. 7. To reduce the influence of noise, the coordinates of the beam center $\mathrm{X}, \mathrm{Y}$ and of the second moment $\mathrm{Q}$ are estimated from a sample of no less than 32 current pulses, then the results are averaged. Fig. 6 shows the beam parameters X, Y and $Q$ at the ELA EPOS exit, observed for two hours of one of the accelerator runs.

The above-described channels and methods together with the channels monitoring the beam energy [6] and position on the target [7] provide an effective operation of highcurrent electron linacs

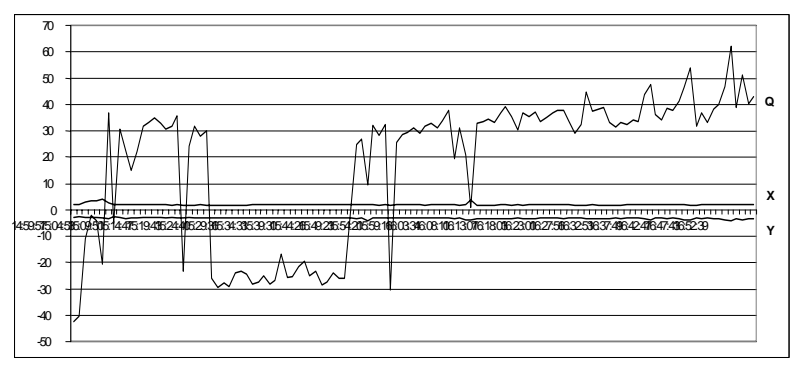

Figure 6: Temporal variation of beam center $(\mathrm{X}, \mathrm{Y}, \mathrm{mm})$ and second moment $(\mathrm{Q}=\sigma \mathrm{x} 2-\sigma \mathrm{y} 2, \mathrm{~mm} 2)$ coordinates at the EPOS exit (beam current $\mathrm{I}=0.71 \mathrm{~A}$, energy $\mathrm{E}=22$ $\mathrm{MeV})$. The measurements were performed for two hours at a one-minute interval

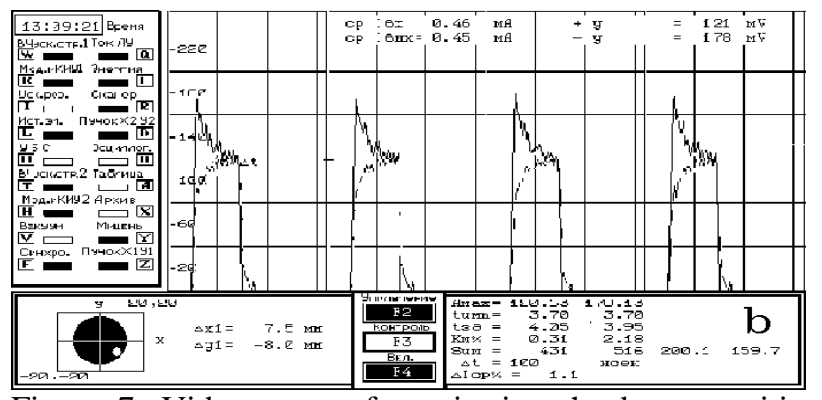

Figure 7: Videograms of monitoring the beam position on the CRT screen of the ELA operator

\section{REFERENCES}

[1] V.N. Boriskin, V.A. Gurin, L.V. Reprintsev et al., "Beam position monitoring channel in a high-current ELA", Proc. of Meeting on Charged Particle Accelerators, Protvino, 1996, v.1, pp.374-377(in Russian).

[2] S.J. Russell et al., "Characterization of Beam Position Monitors for Measurement of Second Moment", Proc. PAC95, pp.2580-2582.

[3] L.V. Reprintsev, "On measurements of charged particle beam parameters by the electromagnetic induction method". VANT, ser. TFE, is.2(4), Kharkov, KFTI, 1979, pp.36-37.

[4] D. Jackson, Classical electrodynamics (Russ. transl.) Mir publ., 1965.

[5] Yu.I. Akchurin, V.N. Boriskin, N.N. Bakhmetyev et al., "A system for monitoring the technological ELA". VANT, ser. YaFI, is.34, Kharkov, pp.55-57

[6] V.N. Boriskin, A.E. Tolstoy, V.I. Uvarov et al., "Automatic Control of the Electron Energy in Technological Electron Linear Accelerators", Digest of the XIV Meeting on the Accelerated Particles, Protvino, Russia, 1994, vol.2, pp. 97-98

[7] V.N. Boriskin, A.N. Savchenko, V.I. Tatanov, Monitoring of the Electron Beam Position in Industrial Linacs., Proc. PAC99, NY, 1999 